In der Rubrik "Literatur kompakt" werden die wichtigsten Originalarbeiten aus der internationalen Fachliteratur referiert.

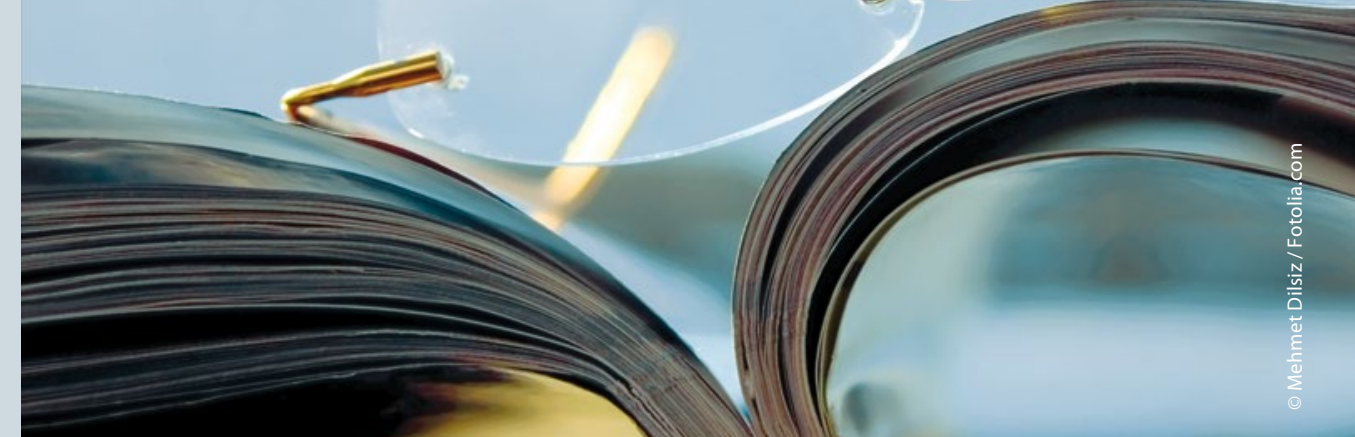

\section{Otitis media: keine verkürzte Antibiose bei Babys}

\begin{abstract}
Gute Gründe sprechen einem US-Ärzteteam zufolge gegen die Abkürzung einer antibiotischen Therapie bei Babys mit Otitis media. In der randomisierten, placebokontrollierten Studie schlug die fünftägige Behandlung deutlich schlechter an als das Standardregime über zehn Tage, und die Kinder benötigten letztlich mehr Antibiotika pro Erkältungssaison.
\end{abstract}

\begin{abstract}
Dising ie Forscher um Dr. Alejandro Hoberman von der Universität Pittsburgh hatten insgesamt 520 kleine Patienten im Alter zwischen sechs und 23 Monaten für die Intention-to-treat-Analyse ausgewählt. Die Otitis-media-Diagnose beruhte auf dem Vorliegen dreier Kriterien: einer Symptomstärke von mindestens 3 von maximal 14 Punkten auf der AOM-SOS-(Acute Otitis MediaSeverity of Symptoms)-Skala, Flüssigkeit im Mittelohr und leichte bis mittelgradige Vorwölbung des Trommelfells begleitet von deutlicher Rötung oder Ohrenschmerzen. Die Kinder wurden per Losverfahren entweder dem Standardregime (zehn Tage Amoxicillin-Clavulansäure) oder der verkürzten Therapie (Einnahme ebendieses Antibiotikums
\end{abstract}

\section{US-Ärzte plädieren für eine zehntägige Antibioti-} ka-Therapie bei Kleinkindern mit Otitis media

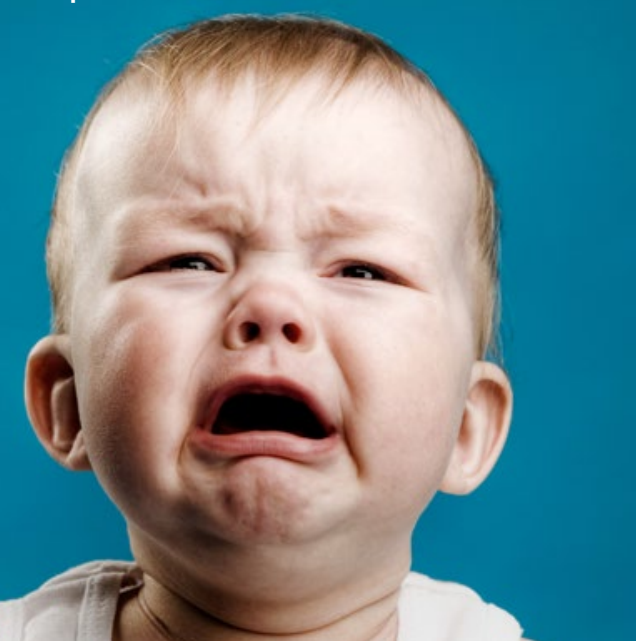

über fünf Tage gefolgt von fünf Tagen unter einem gleich aussehenden, gleich riechenden und gleich schmeckenden Placebo) zugeteilt.

In der Behandlungsphase wurden von den Eltern täglich die AOM-SOS-Scores ihres Kindes ermittelt. Am 12. bis 14. Tag nach Therapiebeginn folgte dann eine Praxisvisite beim Arzt. Erneute Visiten fanden über die gesamte Erkältungssaison (1. Oktober bis 31. Mai) hinweg alle sechs Wochen sowie noch einmal abschließend im darauffolgenden September statt.

Der primäre Endpunkt, das klinische Therapieversagen, trat bei $34 \%$ der Kinder in der Fünf-Tage-Gruppe ein, dagegen nur bei $16 \%$ der Standardgruppe. Als Versagen hatte man eine Symptomverschlechterung, eine Verschlechterung des otoskopischen Befunds oder die ausbleibende Heilung definiert.

Bei einem absoluten Unterschied von 17 Prozentpunkten (basierend auf nicht gerundeten Werten) und einem 95\%-Konfidenzintervall (KI) zwischen 9 und 25 war das Kriterium für Nicht-Unterlegenheit der Kurzzeittherapie nicht erfüllt; laut Hoberman und Kollegen wäre hierfür eine KI-Obergrenze von 10 Prozentpunkten erforderlich gewesen. Wie die Forscher betonen, traten außerdem nur in der Kurzzeitgruppe AOMSOS-Werte $>8$ auf.
Die Ergebnisse der Per-ProtokollAnalyse fielen im Hinblick auf den primären Endpunkt ähnlich aus.

Die mittlere Symptomstärke lag in der Kurzzeitgruppe bei 1,61 an Tag 6 bis 14 und bei 1,89 an Tag 12 bis 14. In der Gruppe mit Standardtherapie betrugen die entsprechenden Werte 1,34 bzw. 1,20. Signifikant war dabei nur die Differenz im Zeitabschnitt zwischen Tag 12 und 14. Den Forschern zufolge befanden sich in der Fünf-Tage-Gruppe zudem deutlich weniger Kinder, bei denen die Symptomstärke im Therapieverlauf um mehr als $50 \%$ abgenommen hatte ( $80 \%$ vs. $91 \% ; \mathrm{p}=0,003)$.

Keine signifikanten Unterschiede fand man dagegen bei den Hauptnebenwirkungen der Antibiotikatherapie: 29\% (verkürzte Therapie) bzw. 30 \% (Standardtherapie) hatten Durchfall entwickelt, unter Windelsoor litten $34 \%$ bzw. $33 \%$.

Rezidive ereigneten sich in beiden Gruppen in etwa gleich häufig; dabei waren jeweils vor allem die Kinder betroffen, die zu Therapieende noch Reste von Flüssigkeit im Mittelohr aufwiesen, unabhängig davon, ob man die Therapie als erfolgreich oder erfolglos eingestuft hatte.

Fazit: Letztlich, so Hoberman und sein Team, seien die Unterschiede groß genug, um zu zeigen, dass das Standardregime der verkürzten Therapie an Wirksamkeit überlegen sei. Die Ergebnisse würden jedoch nur für die untersuchte Altersgruppe unter zwei Jahren gelten und ließen sich nicht auf ältere Kinder übertragen.

Elke Oberhofer

Hoberman A et al. Shortened Antimicrobial Treatment for Acute Otitis Media in Young Children. N Engl J Med 2016; 375: 2446-56 